\title{
ANALYSIS OF MAGNETOHYDRODYNAMIC JEFFERY-HAMEL FLOW IN A CONVERGENT-DIVERGENT CHANNEL USING $C u$-WATER NANOFLUID
}

\author{
Ashik Chandra Das*, Qazi Novera Tansue Nasa and Md. Sarwar Alam \\ Department of Mathematics, Jagannath University, Dhaka, Bangladesh
}

Received: 28 April 2021

Accepted: 16 June 2021

\begin{abstract}
The present study focused on the entropy generation as well as the heat transfer rate and velocity profiles of a nanofluid of the Jeffery-Hamel flow, especially for convergent-divergent channels. First, the governing dimensional partial differential equations have been transformed into a system of non-dimensional ordinary differential equations. The Power series method has been used to solve these non-dimensional governing equations and the Hermite-Pade approximation has been applied for analyzing them. The effect of various physical parameters such as channel angle, Reynolds number, Hartmann number, nanoparticle solid volume frictions and Eckert number have been investigated for the velocity profiles, heat transfer and entropy generation. Here, $\mathrm{Cu}$ has been used as the solid nanoparticle and water has been used as the base fluid. It is interesting to remark that the entropy generation of the whole system increased at the two walls and a significant effect could be noticed on the heat transfer rate and velocity profiles.
\end{abstract}

Keywords: Convergent-divergent channel, Cu-nanoparticles, Hermite-Pade approximation, Jeffery-Hamel flow, MHD, Power series method.

$\begin{array}{cl}\text { Nomenclature: } & \\ f & \text { Fluid } \\ s & \text { Solid particle } \\ n f & \text { Nanofluid } \\ \mathrm{Ha} & \text { Hartmann number } \\ \mathrm{Re} & \text { Reynolds number } \\ \mathrm{Ec} & \text { Eckert number } \\ \mathrm{Pr} & \text { Prandtl number } \\ \mathrm{Ns} & \text { Entropy generation }\end{array}$

$\begin{array}{ll}\alpha & \text { Channel angle } \\ \rho & \text { Density } \\ \eta & \text { Dimensionless angle } \\ \mu & \text { Dynamic viscosity } \\ v & \text { Kinematic viscosity } \\ \phi & \text { Solid volume friction } \\ \beta & \text { Dimensionless temperature } \\ \varphi & \text { Any angle }\end{array}$

\section{INTRODUCTION}

The dilute suspension of two particles is known as nanofluid. One of them is a solid nanoparticle and the other is a base fluid. Different type of solid nanoparticles may be used like $\mathrm{Cu}, \mathrm{Al}_{2} \mathrm{O}_{3}, \mathrm{TiO}_{2}, \mathrm{ZnO}$ and so on. These particles are mainly nanosized (less than $100 \mathrm{~nm}$ ). Besides these solid nanoparticles behave as good conductors of heat. They also enable the base fluid to enhance its thermal properties. In this case, water, ethylene glycol, oil etc. may be used as the base fluid which is available in (Das et al., 2007). But the word nanofluid was first introduced by (Choi, 1995). After that, a good number of researchers have been successfully drawn their attention to nanofluid. This is the reason that the applications of nanofluid have become an interesting issue in recent times. Nanofluid is used in many industrial and engineering processes like environmental, mechanical, biomechanical, aerospace, chemical and civil engineering. The flux of the rivers and canals is also a splendid application of nanofluid. Another real-life example of nanofluid is the blood flow in the human body.

The classical Jeffery-Hamel flow is considered as the flow of a two-dimensional viscous incompressible fluid in a channel. This channel may be parallel or convergent-divergent. In the convergent-divergent channel, the two non-parallel walls are driven by a source or sink and are separated by a fixed angle. It was briefly described in (Hamadiche et al., 1994). The concept of the classical Jeffery-Hamel flow had come to the attention of the researchers since the first published study of (Jeffery, 1915) and (Hamel, 1916). Since then numerous studies have been done on the classical Jeffery-Hamel flow using nanofluid. Later, it can be found that there exists a similarity solution between the solutions of this flow and the Navier-Stokes equations for two dimensionless parameters. These two dimensionless parameters are mainly the Reynolds number and the channel angular width. (Fraenkel, 1962) then studied the laminar flow in symmetrical channels with slightly curved walls using 
the Jeffery-Hamel solutions for flow between plane walls. It was found in this study that for viscous source or sink flow between straight walls of any plane, the solutions of the Jeffery-Hamel flow were mostly the same. So, in his analysis, he regarded the Jeffery-Hamel solutions as the leading term of a series solution for the velocity field of the flow.

Recently, (Jeong et al., 2008) has investigated that in fundamental concepts of spreading and adhesion on a solid surface, nanofluid behaves electrically like classical liquid. The compressible Navier-Stokes equations are derived for the slip boundary conditions which is available in (Aoki, 2017). He has studied it from the Boltzmann equation based on the Chapman-Enskog solution. (Sobey and Drazin, 1986) have also used analytical, experimental and numerical methods to investigate the instabilities and bifurcations of twodimensional channel flows. They have found a rich and unexpected structure for Reynolds numbers of less than a few hundred to the solutions of the Navier stoke equation. Moreover, (Moradi et al., 2015) have studied the non-linear Jeffery-Hamel flow in a fluid for the effect of heat transfer. Three different types of nanoparticles are used in their study. They have observed that the effect of solid nano-particle volume friction on the fluid flow parameters and the heat transfer are more stable. Turbulent friction and heat transfer behaviours of dispreads fluids have been investigated by (Pak and Cho, 1998). A circular pipe has been used in their experiment with alumina $\left(\mathrm{Al}_{2} \mathrm{O}_{3}\right)$ and titanium dioxide $\left(\mathrm{TiO}_{2}\right)$ have been used as the solid particles having diameters of $13 \mathrm{~nm}$ and $27 \mathrm{~nm}$, respectively. The results of their study demonstrate that the convective heat transfer coefficient of the dispersed fluid is smaller than the pure water under the condition of constant velocity.

Presently, many researchers have given their attention to develop the analysis of nanofluid. Different types of study have been being continued. In recent times, the combined free and forced convection MHD flow in a rotating channel with a perfectly conducting wall have been studied by (Seth and Singh, 2008). They have observed that if the fluid is electrically conducting then the current induces Joule heating. It is also found that this induced Joule heating depends on the strength of the magnetic field. The entropy generation minimization for forced-free convective heat transfer due to viscosity effects and temperature gradient in a fluid was studied by (Bejan, 1996). Recently, (Alam et al., 2017) have studied the entropy generation of steady MHD

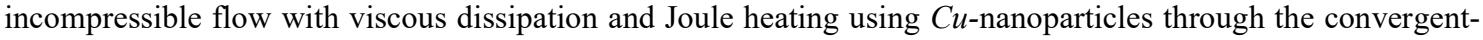
divergent channel. In their study, the remarkable result was that the entropy generation of the system increased at the two walls. They also found that along the centerline of the channel the heat transfer irreversibility and the fluid friction irreversibility were dominant there.

The present study is focused on the effect of different thermophysical properties on entropy generation as well as on the velocity and temperature profiles considering viscous dissipation and Joule heating in the convergentdivergent channel. The velocity profiles, temperature distributions and entropy generation, as well as the rate of heat transfer, have been discussed graphically for the variation of channel angle $\alpha$, Reynolds number Re, Hartman number $\mathrm{Ha}$, Eckert number $E c$ and nanoparticle volume friction $\phi$. The Hermite-Pade approximation (HPA) has been applied to solve the power series. The bifurcation diagrams of the skin friction against divergent channel angle and Reynolds number have also been presented here.

\section{DERIVATION OF MATHEMATICAL EQUATION}

In this investigation, a steady two-dimensional incompressible laminar flow of $\mathrm{Cu}$-water nanofluid has been considered which places a source or sink in two non-parallel walls. The angle between the two walls is $2 \alpha$ which is shown in Figure 1. In this case, a cylindrical coordinate system $(r, \varphi, z)$ has been used. Here it is assumed that the velocity is purely radial. This radial velocity depends on $r$ and $\varphi$. This shows that the flow parameter receives no change in the $\mathrm{z}$ direction. Markedly, it is a presumption that an external magnetic field has been acted vertically downward to the top wall. Let the domain of the experimented flow be $-|\alpha|<\varphi<|\alpha|$.

So the semi angle of the channel will be $\alpha$. Now, considering Joule heating and viscous dissipation the continuity equation, Navier-Stokes equation and the energy equation are,

$$
\begin{aligned}
& \frac{\rho_{n f}}{r} \frac{\partial}{\partial r}(r u)=0 \\
& u \frac{\partial u}{\partial r}=-\frac{1}{\rho_{n f}} \frac{\partial p}{\partial r}+v_{n f}\left(\frac{\partial^{2} u}{\partial r^{2}}+\frac{1}{r} \frac{\partial u}{\partial r}+\frac{1}{r^{2}} \frac{\partial^{2} u}{\partial \varphi^{2}}-\frac{u}{r^{2}}\right)-\frac{\sigma_{n f} B_{0}^{2}}{\rho_{n f} r^{2}} u \\
& \frac{1}{\rho_{n f} r} \frac{\partial p}{\partial \varphi}-\frac{2 v_{n f}}{r^{2}} \frac{\partial u}{\partial \varphi}=0
\end{aligned}
$$


$u \frac{\partial T}{\partial r}=\frac{\kappa_{n f}}{\left(\rho c_{p}\right)_{n f}}\left(\frac{\partial^{2} T}{\partial r^{2}}+\frac{1}{r} \frac{\partial T}{\partial r}+\frac{1}{r^{2}} \frac{\partial^{2} T}{\partial \varphi^{2}}\right)+\frac{\mu_{n f}}{\left(\rho c_{p}\right)_{n f}}\left(4\left(\frac{\partial u}{\partial r}\right)^{2}+\frac{1}{r^{2}}\left(\frac{\partial u}{\partial \varphi}\right)^{2}\right)+\frac{\sigma_{n f} B_{0}{ }^{2}}{\left(\rho c_{p}\right)_{n f} r^{2}} u^{2}$

Here it is assumed that the flow is symmetrically radial. So the velocity field has been turned into the form $v=[u, 0,0]$ where $u$ is the function of both $\mathrm{r}$ and $\varphi$. Then the volumetric flow rate through the channel is

$Q=\int_{-\alpha}^{\alpha} u r d \varphi$

Boundary conditions for the problem are as follows,

$u=0, T=T_{\alpha}$ at $\varphi= \pm \alpha$

In the above equations, the velocity components and temperature of the base fluid are $u$ and $T$ respectively. Also $B_{0}, \mathrm{p}, \rho_{n f}, \mu_{n f}, \sigma_{n f}, v_{n f}$ represent the electromagnetic induction, the fluid pressure, the effective density, the effective dynamic viscosity, the electrical conductivity and the kinematic viscosity of the nanofluid respectively and the variations of these physical parameters are given as (Aminossadati and Ghasemi, 2009),

$$
\begin{aligned}
& \rho_{n f}=\rho_{f}(1-\phi)+\rho_{s} \phi, \quad \mu_{n f}=\frac{\mu_{f}}{(1-\phi)^{2.5}}, \quad v_{n f}=\frac{\mu_{n f}}{\rho_{n f}} \\
& \frac{\sigma_{n f}}{\sigma_{f}}=1+\left[3\left(\frac{\sigma_{s}}{\sigma_{f}}-1\right) \phi /\left(\left(\frac{\sigma_{s}}{\sigma_{f}}+2\right)-\left(\frac{\sigma_{s}}{\sigma_{f}}-1\right) \phi\right)\right]
\end{aligned}
$$

Now the corresponding heat capacity and the effective thermal conductivity of the nanofluids are,

$$
\kappa_{n f}=\kappa_{f} \frac{\kappa_{s}+2 \kappa_{f}-2 \phi\left(\kappa_{f}-\kappa_{s}\right)}{\kappa_{s}+2 \kappa_{f}+\phi\left(\kappa_{f}-\kappa_{s}\right)}, \quad\left(\rho c_{p}\right)_{n f}=(1-\phi)\left(\rho c_{p}\right)_{f}+\phi\left(\rho c_{p}\right)_{s}
$$

Here $\phi$ is the nanoparticle volume friction of the nanoparticles. $\kappa_{s}$ and $\kappa_{f}$ represent the thermal conductivities of solid particle and the base fluid respectively. Also $\left(\rho c_{p}\right)_{n f}$ is the heat capacity of the nanofluid. The thermophysical properties of the nanoparticle and the base fluid are given in Table 1.

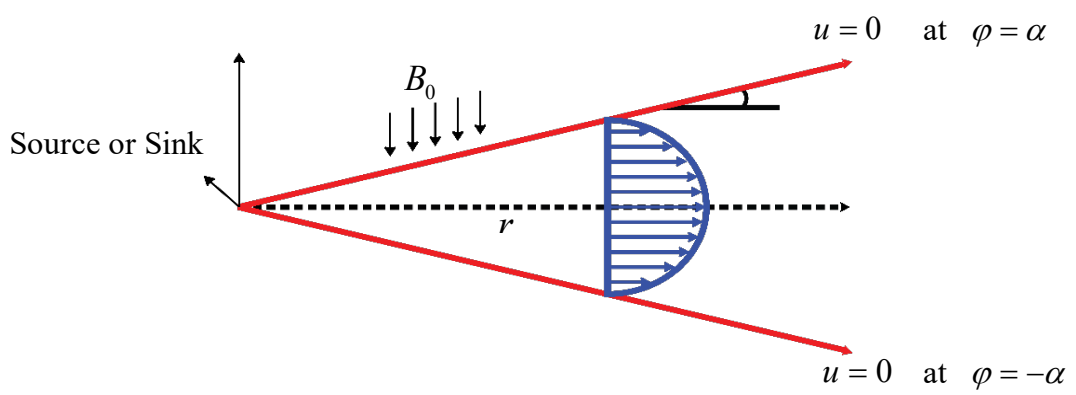

Figure 1: Diagram of the flow problem.

Table 1: Thermophysical properties of the $C u$-nanoparticle and the water (Das et al., 2005).

\begin{tabular}{ccc}
\hline \hline Physical properties & $C u$ & Water \\
\hline \hline$\rho\left(\mathrm{kg} / \mathrm{m}^{3}\right)$ & 8933 & 997.1 \\
$c_{p}(\mathrm{~J} / \mathrm{kgK})$ & 385 & 4179 \\
$\kappa(W / m K)$ & 401 & 0.613 \\
$\sigma(\Omega m)$ & $59.6 \times 10^{6}$ & 0.05 \\
\hline \hline
\end{tabular}

If it requires $Q \geq 0$, then $\alpha>0$, the flow is diverging from a source at $r=0$.

Let the stream function be $\psi=\psi(r, \varphi)$, then it becomes 
$\frac{\partial \psi}{\partial r}=0, \frac{\partial \psi}{\partial \varphi}=u r$

Now the new non-dimensional variables have been established,

$\eta=\frac{\varphi}{\alpha}, F(\eta)=\frac{\psi(\varphi)}{Q}, \theta(\eta)=\frac{T}{T_{\alpha}}$

The reduced ordinary differential equation of the governing equations (2) - (4) can be written in the following form,

$$
\begin{aligned}
& F^{(\mathrm{iv})}+2 \alpha \operatorname{Re} A(1-\phi)^{2.5} F^{\prime} F^{\prime \prime}+\left(4-(1-\phi)^{2.5} D H a^{2}\right) \alpha^{2} F^{\prime \prime}=0, \\
& \theta^{\prime \prime}+\frac{B E c \operatorname{Pr}}{C(1-\phi)^{2.5}}\left[4 \alpha^{2} F^{\prime 2}+\left(F^{\prime \prime}\right)^{2}+(1-\phi)^{2.5} D H a^{2} \alpha^{2} F^{\prime 2}\right]=0
\end{aligned}
$$

The corresponding boundary conditions have been reduced to the form,

$F(-1)=-1, \quad F(1)=1, F^{\prime}( \pm 1)=0, \quad \theta( \pm 1)=1$

$\operatorname{Re}$ is the Reynolds number and it is defined as $\operatorname{Re}=\frac{Q}{v_{f}}$. Furthermore, $\operatorname{Pr}=\frac{\left(\mu c_{p}\right)_{f}}{\kappa_{f}}$ is the Prandtl number, $E c=\frac{U_{\max }^{2}}{\left(c_{p}\right) T_{\alpha}}$ is the Eckert number and $H a=\sqrt{\frac{\sigma_{f} B_{0}{ }^{2}}{\rho_{f} v_{f}}}$ is the Hartman number. And some more assumed constants are

$$
\begin{aligned}
& A=(1-\varphi)+\frac{\rho_{s}}{\rho_{f}} \phi, \quad B=(1-\varphi)+\frac{\left(\rho c_{p}\right)_{s}}{\left(\rho c_{p}\right)_{f}} \phi, \quad C=\frac{\kappa_{s}+2 \kappa_{f}-2 \phi\left(\kappa_{f}-\kappa_{s}\right)}{\kappa_{s}+2 \kappa_{f}+\phi\left(\kappa_{f}-\kappa_{s}\right)} \\
& D=1+\left[3\left(\frac{\sigma_{s}}{\sigma_{f}}-1\right) \phi /\left(\left(\frac{\sigma_{s}}{\sigma_{f}}+2\right)-\left(\frac{\sigma_{s}}{\sigma_{f}}-1\right) \phi\right)\right]
\end{aligned}
$$

\section{SERIES ANALYSIS}

In this investigation, the equations have been solved using the series solution method. These series have been analysed by considering different non-linear terms and then the equations are also expressed through a parameter. For solving these, the power series expansion in terms of the parameter $\alpha$ have been considered first. For initially performing this problem, Equation (10) and Equation (11) can be written in the non-linear form for stream function and temperature profile. These non-linear terms are as follows,

$$
F(\eta)=\sum_{i=0}^{\infty} F_{i}(\eta) \alpha^{i}, \quad \theta(\eta)=\sum_{i=0}^{\infty} \theta_{i}(\eta) \alpha^{i} \quad \text { as } \quad|\alpha|<1
$$

Now by substituting Equation (13) into Equation (10) and Equation (11) and using the corresponding boundary conditions given in Equation (12), the dimensionless governing equations are then solved into a series solution. First of all, the coefficients of powers of $\alpha$ have been equated in both sides of the equation. We have then computed it for the series of the stream function $F(\eta)$ and temperature $\theta(\eta)$. For solving this more accurately, the programming software MAPLE has been used. By using this software, the first 12 coefficients for these series in terms of $\alpha, \phi, \operatorname{Re}, H a, E c, \operatorname{Pr}, A, B, C, D$ have been computed. The different non-dimensional parameters are also expressed here.

The expressions of shear stress and skin friction are as follows,

$$
\tau_{w}=\mu_{n f}\left(\frac{1}{r} \frac{\partial u}{\partial \varphi}\right) \quad \text { and } \quad c_{f}=\frac{\tau_{w}}{\rho_{f}\left(U_{\max }\right)^{2}}
$$


Using the non-dimensional transformation Equation (9) in the above Equation (14), the reduced form is obtained from Equation (14).

$c_{f}=\frac{1}{\operatorname{Re}(1-\phi)^{2.5}} F^{\prime \prime}(1)$

\section{HERMITE PADE APPROXIMANT}

Hermite-Pade approximant is a method of solution of a non-linear system of equation. This method is first introduced by (Hermite, 1893) and (Padé, 1892). In this study, conditions of criticality along with earlier mentioned irreversibility in the system by the Hermite-Pade method have been computed accurately. This approximation method is as follows,

For $|\alpha|<1$, S be the partial sum of the series. By applying rapid polynomial approximation, desired sum can be obtained.

$S_{N-1}(\alpha)=\sum_{n=0}^{N-1} a_{n} \alpha^{n}$

Let $d$ be any positive integer and $(\mathrm{d}+1)$ tuple of polynomials then Hermite-Padé form is

$\operatorname{deg} P_{N}^{[0]}+\operatorname{deg} P_{N}^{[1]}+\ldots+\operatorname{deg} P_{N}^{[d]}+d=N$, if

$\sum_{i=0}^{d} P_{N}^{[i]}(\alpha) S_{i}(\alpha)=O\left(\alpha^{N}\right) \quad$ as $\quad|\alpha|<1$

where $S_{0}(\alpha), S_{1}(\alpha), \ldots, S_{d}(\alpha)$ different forms of a single series or independent series.

After solving Equaton (17) and (18) got the polynomials $P_{N}^{[i]}$ which are determined by their coefficient. The unknowns in the Equation (18) is

$\sum_{i=0}^{d} \operatorname{deg} P_{N}^{[\mathrm{i}]}+d+1=N+1$

Here it is confirmed by the equation that the coefficient matrix related to this system is square.

Now the left side of the Equation (18) has been expanded in power of $\alpha$ and then equated $N$ equations of the system equal to zero. In this case, a system of linear homogeneous equations can be found. To perform the next step of the solution, some kind of normalization is needed to calculate the coefficients of the Hermite-Padé polynomials such as,

$P_{N}^{[i]}(0)=1$ for some integer $0 \leq i \leq d$

$S_{0}(\alpha), S_{1}(\alpha), \ldots, S_{d}(\alpha)$ are the first $N$ coefficients of the series, which are required for the calculation of the Hermite-Padé polynomials. For that, the linear equations have been solved by Gaussian elimination or GaussJordan elimination. In this case, if the singularity is of an algebraic type, then the exponent $\delta$ may be approximated by

$\delta_{N}=d-2-\frac{P_{N}^{[d-1]}\left(\alpha_{c, N}\right)}{D P_{N}^{[d]}\left(\alpha_{c, N}\right)}$

Here two unique moderately approximants HODA (High-order differential approximant) and HPDA (Highorder partial differential approximants) have been carefully introduced by (Khan, 2002) and (Rahman, 2004) respectively. With the above-mentioned methods, a particular algebraic approximant have been used additionally which was introduced by (Drazin and Tourigney, 1996).

\section{ANALYSIS OF IRREVERSIBILITY}

In this century, the world is going to face a large problem as people are using non-renewable energy without any limit. This limitless use of energy causes much wastage too. The result goes this renewable energy is being lost day by day from the world. If it is continuing, it will be impossible to live in the world. So, it is one of the major concern of the engineers and the researchers is to find the methods that can control the wastage of useful energy. 
It is interesting that there is a significant influence of MHD on this system. A great example of this system is that in a convergent-divergent channel with the isothermal walls, the properties of flow in presence of a magnetic field and viscous dissipation are irreversible. At the solid boundaries, the temperature in equilibrium conditions arises because of the exchange of energy and momentum within the fluid. This energy produces continuous entropy generation. For the fully developed flow, the volumetric entropy generation rate in cylindrical coordinates is given as,

$E_{G}=\frac{\kappa_{n f}}{T_{\alpha}^{2}}\left(\frac{d T}{d \varphi}\right)^{2}+\frac{\mu_{n f}}{T_{\alpha}}\left(\frac{d u}{d \varphi}\right)^{2}+\frac{\sigma_{n f} B_{0}{ }^{2}}{T_{\alpha}} u^{2}$

Here, entropy generation is determined by the summation of three terms. The first term of the right side of Equation (21) is the irreversibility due to heat transfer. The second and third terms of the right side of the above Equation (21) are the irreversibility due to viscous dissipation and the local entropy generation due to the effect of the magnetic field, respectively. The non-dimensional form of the entropy generation number can be reduced as,

$N_{s}=\frac{\alpha^{2} E_{G}}{\kappa_{f}}=\frac{\kappa_{n f}}{\kappa_{f}}\left(\frac{d \theta}{d \eta}\right)^{2}+\frac{B \operatorname{Pr} E c}{(1-\phi)^{2.5}}\left(\frac{d^{2} F}{d \eta^{2}}\right)^{2}+B D \operatorname{Pr} E c \alpha^{2} H a^{2}\left(\frac{d F}{d \eta}\right)=N_{1}+N_{2}+N_{3}$

Where, $N_{1}=\frac{\kappa_{n f}}{\kappa_{f}}\left(\frac{d \theta}{d \eta}\right)^{2}, \quad N_{2}=\frac{B \operatorname{Pr} E c}{(1-\phi)^{2.5}}\left(\frac{d^{2} F}{d \eta^{2}}\right)^{2}, \quad N_{3}=B D \operatorname{Pr} E c \alpha^{2} H a^{2}\left(\frac{d F}{d \eta}\right)$

In this paper, the focus of the study is observing the entropy generation for the optimum values of the parameters and discussing the obtained outcomes.

\section{RESULTS AND DISCUSSION}

In the present study, the influence of different parameters of interest in the convergent-divergent channel have been observed for the problem. Entropy generation, temperature distribution and velocity profiles are performed here for the parameters. The parameters which are investigated here are the nanoparticle volume friction $\phi$, magnetic Hartman number $\mathrm{Ha}$, Reynolds number Re, channel angle $\alpha$ and Eckert number Ec. Also, the solid volume friction for $\mathrm{Cu}$-nanoparticles is studied in the range of $0 \leq \phi \leq 0.2$ and the Prandtl number Pr for the base fluid is kept at 6.2 here. The graphical representations of the outcomes are presented and discussed for this study.

\subsection{Stability Analysis}

It is mentioned earlier that in this study the centerline axial velocity and the radial velocity will be investigated as two series in powers of $\alpha, H a, \operatorname{Re}, \phi, A$ and $D$. Here, Table 2 exhibits the convergence of $\alpha_{c}$ for $d=3$ using $N=12$ up to 12 decimal places. This study has been performed for a different amount of the presence of nanoparticle in the nanofluid. The variation in the critical values $\alpha_{c}$ with critical exponent $\delta$ for four different values of solid volume friction $\phi=0.00,0.07,0.14,0.20$. If the present outcome is observed, there is an indication that there is a great influence of the presence of nanofluids in the instability of the flow process. It is seen in the flow that these nanofluids result from early development in the system.

Table 2: Numerical values of critical angles $\alpha_{c}$ and their exponent $\delta$ at $H a=1$ and $\operatorname{Re}=20$.

\begin{tabular}{ccc}
\hline$\phi$ & $\alpha_{c}$ & $\delta$ \\
\hline 0.00 & 0.271769826156 & 0.450319299852 \\
0.07 & 0.210881499718 & 0.507663726998 \\
0.14 & 0.187752358428 & 0.428501987679 \\
0.20 & 0.183265993141 & 0.421205605495 \\
\hline
\end{tabular}

Here, the algebraic approximation method has been applied to our series. By applying this method, the bifurcation graphs of skin friction coefficient against $\alpha$ are obtained. Figure 2 denotes the bifurcation graph of skin friction against $\alpha$ for the $C u$-nanoparticles. It is interestingly noticed from Figure 2 that there is a turning point or a saddle-node bifurcation at $\alpha=\alpha_{c}$. It is also noticeable from the bifurcation diagram that there is a growing effect for the increasing amount of $\mathrm{Cu}$-nanoparticles in the fluid. 
Here two solutions branches for the skin friction for different values of nanoparticle volume friction have been observed. One is for $\alpha<\alpha_{c}$ and the other marginal solution is for $\alpha=\alpha_{c}$. But there is no solution for $\alpha>\alpha_{c}$. It is clear from the above figure that the bifurcation points have become change for various values of $\phi$. The bifurcation points are obtained at $\mathrm{Re}=20, H a=1$. Here, it is also noted that the skin friction coefficient is increased by the solid volume friction.

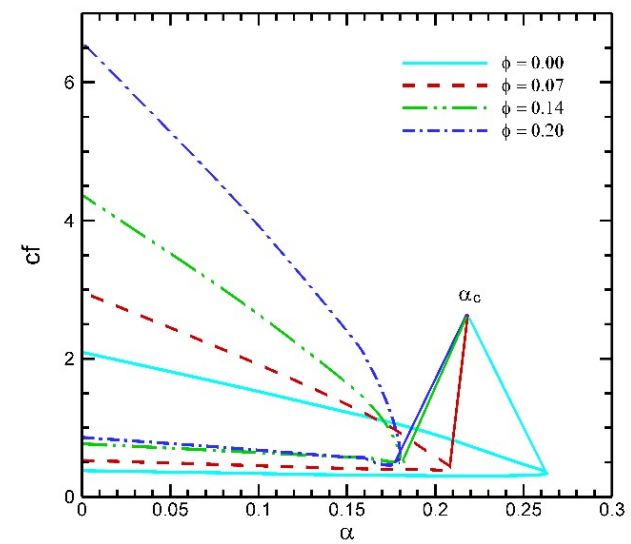

Figure 2: Bifurcation diagram of skin friction coefficient versus divergent channel angle $\alpha$ at $\operatorname{Re}=20, H a=1$ for $C u$-nanofluid.

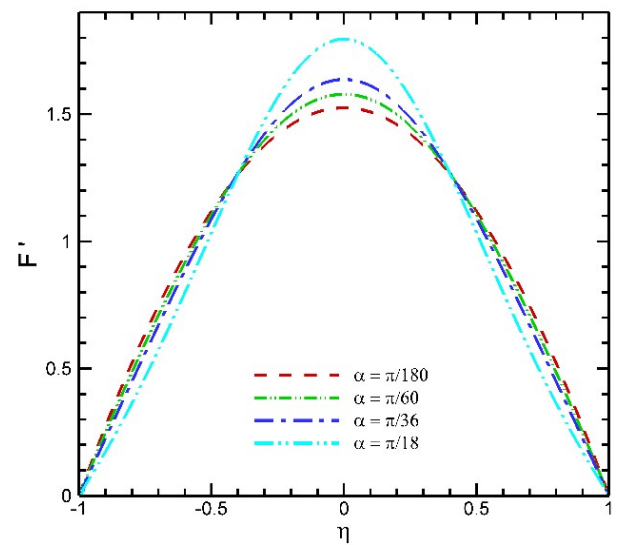

(a)

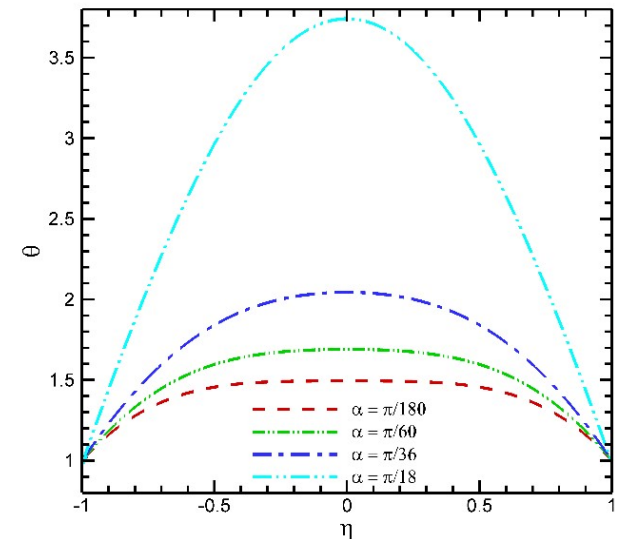

(b)

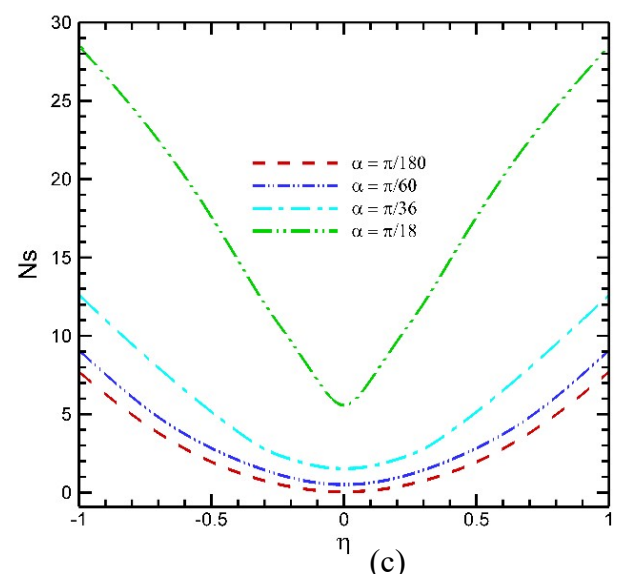

Figure 3: (a) Velocity profile (b) Temperature distribution (c) Entropy generation for different values of $\alpha$ at $\phi=0.07, \operatorname{Re}=20, H a=10, \operatorname{Pr}=6.2, E c=0.1$ 


\subsection{Diverging Channel}

A non parallel channel having a source by which the fluid enters into the channel is known as a diverging channel. The effect of different parameters for the entropy generation as well as velocity profiles and temperature distribution have been discussed for the divergent channel here. Below the key figures, the unique results have come out from the study of the diverging channel.

Figure 3(a) represents the effect of channel angle on velocity profiles for $C u$-nanofluid in the divergent channel. Here it can be noticed that the presence of $C u$-nanoparticles $(\phi=0.07)$ expedites the velocity at the centerline of the channel. It is also observed that a major backflow occurs for the large channel angel in the two walls of the channel. The effect of increasing channel angle is also noticed on temperature distribution for the divergent channel. Figure 3(b) represents the temperature distribution for the increasing channel angle. It is significantly noticed that for the large angle $\pi / 18$, the nanofluid generates the highest temperature values and $\pi / 180$ exhibits the lowest heat transfer rate. There is also seen that at the centerline of the channel, the heat transfer rate reach the highest. It is mentioned in many affiliated textbooks and research papers that the heat transfer property in a flow region is enhanced by the characteristics of nanofluids. The influence of nanofluid in entropy generation is also noticed here. The impact of channel angle on the entropy generation is shown in Figure 3(c). It is interesting to percept here that the entropy generation has a great influence on the channel opening. The increasing channel angle provides a more entropy rate for the $C u$-nanofluid. Here it is seen that in the channel angle $\pi / 18$, the entropy gives the highest rate followed by a small channel angle. But the entropy is high at the two walls more than the centerline of the channel. It is a splendid effect that the lowest channel opening has not only the lowest velocity profiles but also the lowest channel angle exhibits the lowest temperature and entropy generation. The lowest channel angle is kept $\pi / 180$ in this study. It is interesting to notice that the outcomes of the increasing channel angle has also an increasing effect on this study.

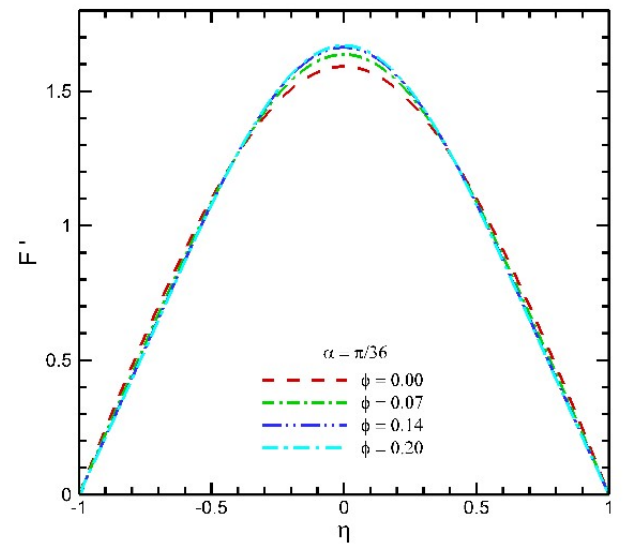

(a)

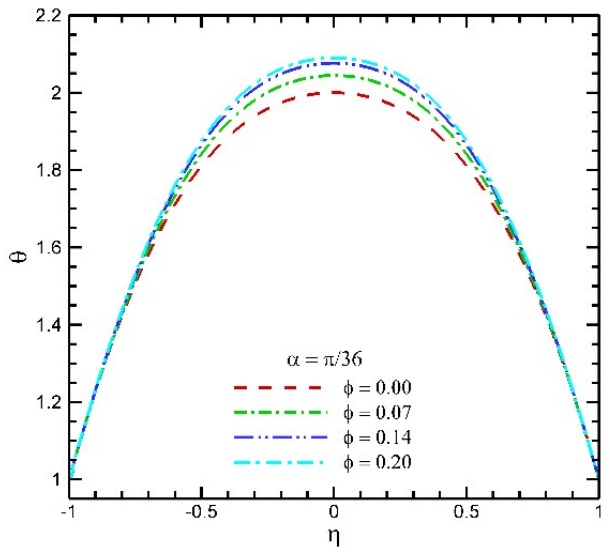

(b)

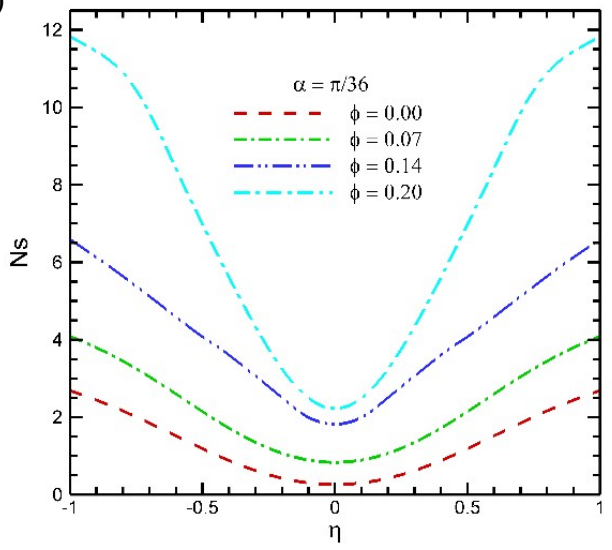

(c)

Figure 4: (a) Velocity profile (b) Temperature distribution (c) Entropy generation for different values of $\phi$ at $\alpha=\pi / 36, \operatorname{Re}=20, H a=10, \operatorname{Pr}=6.2, E c=0.1$ 
Figure 4(a) represents the velocity profiles with the variations of nanoparticle volume friction $\phi$. It is seen from the figure that the presence of nanoparticles has a great influence on the velocity profiles. Without any nanoparticles which means only base fluid water has the lowest velocity rate. Perhaps the increasing amount of nanoparticles in the nanofluid produces high velocity in the channel. The effect of nanoparticles in the temperature distribution is presented in Figure 4(b). A noticeable effect for nanoparticles volume friction is found in this temperature distribution. The temperature rises high at the centerline of the channel. It is also significant in Figure 4(b) that at the two walls of the channel, the temperature remains almost the same. The entropy generation for the variations of nanoparticles volume is plotted in Figure 4(c). It is interesting to notice here that the velocity profiles and temperature distribution $\phi=0.20$ gives the highest entropy.

The effects of Reynolds number on velocity profile, temperature distribution and entropy generation are shown in Figure 5(a)-5(c) respectively. With the fixed nanoparticle volume friction and channel angle, the velocity profile rises high with the increasing Reynolds number. The same changes can be noticed in Figure 5(b). The temperature distribution for different Re shows different outcomes here. The highest Re results at the highest temperature for the divergent channel. But the temperature of the fluid near the non-parallel walls persists identical. There is also an opposite influence of Re on the entropy generation for the $\mathrm{Cu}$-based nanofluid. From Figure 5(c) it is clear that $\mathrm{Re}=10$ gives the highest entropy for the nanofluids. A significant change can be noticed that at the centerline, the entropy rates for $\mathrm{Re}=30$ is the fastest followed by the others.

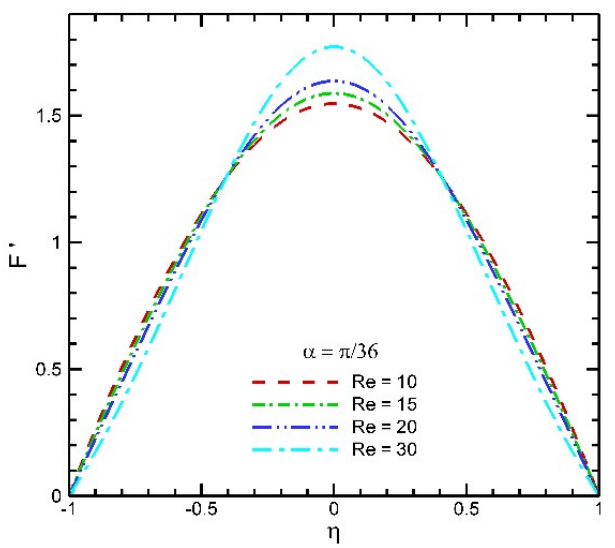

(a)

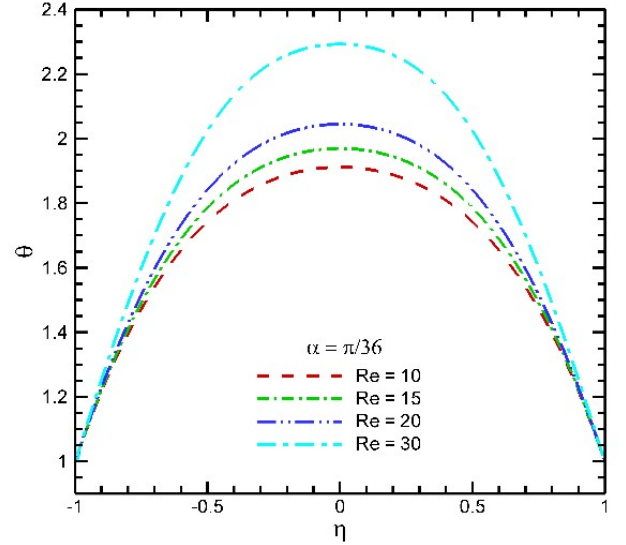

(b)

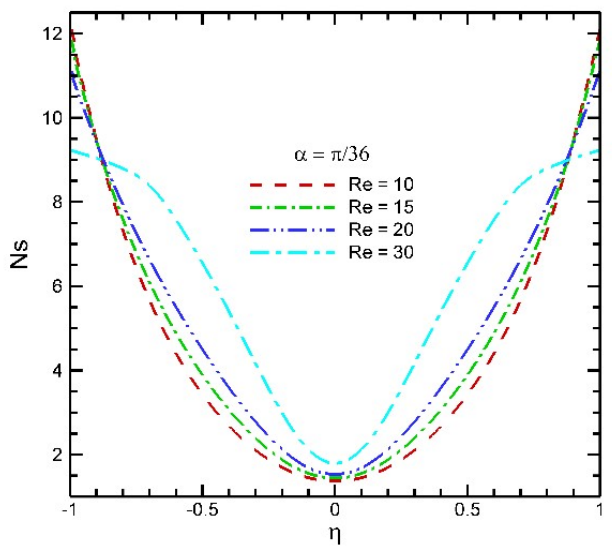

(c)

Figure 5: (a) Velocity profile (b) Temperature distribution (c) Entropy generation for different values of Re at $\alpha=\pi / 36, \phi=0.07, H a=10, \operatorname{Pr}=6.2, E c=0.1$ 


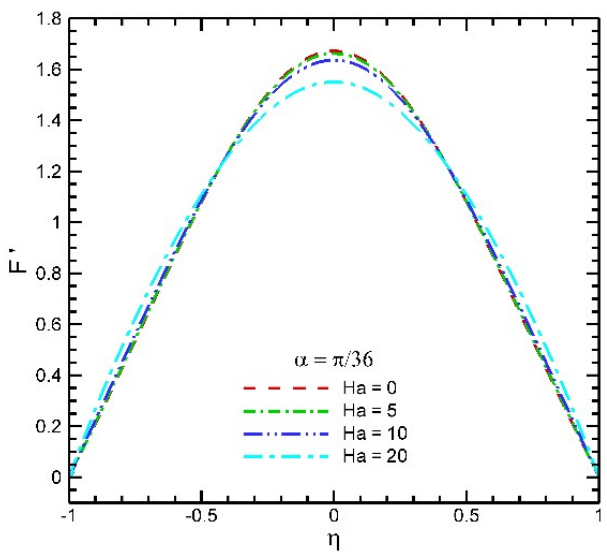

(a)

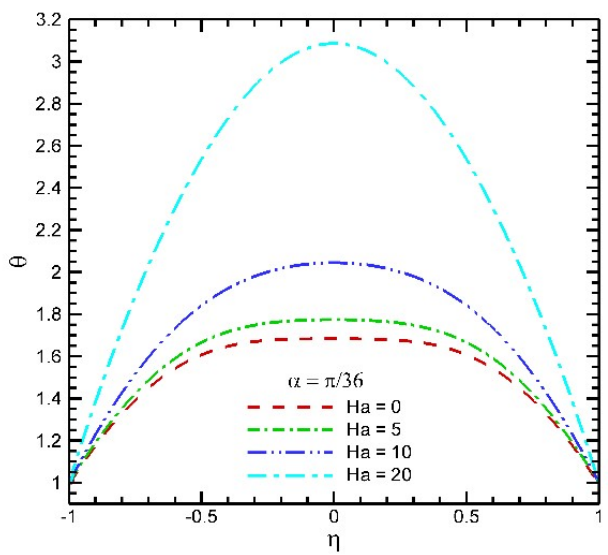

(b)

Figure 6: (a) Velocity profile (b) Temperature distribution for different values of $H a$ at $\alpha=\pi / 36, \phi=0.07, \operatorname{Re}=20, \operatorname{Pr}=6.2, E c=0.1$

Figure 6(a) and 6(b) represents the velocity profiles and temperature distribution respectively. In the effect of Hartman number, $\mathrm{Ha}$ has a conversed effect on the velocity profiles. For the divergent channel with a fixed channel angle and flow rate, the velocity profiles have reduced for the increasing Hartman number. It is seen in the above figure that for $H a=0$, the velocity reaches the highest level at the centerline and then for different values of $\mathrm{Ha}$ greater than 0 show their result. The temperature distribution for the increasing $\mathrm{Ha}$ is also plotted in Figure 6(b). It is interestingly noticed that with the presence of an external magnetic field, the rate of heat transfer increases rapidly due to a faster rate of $\mathrm{Ha}$.

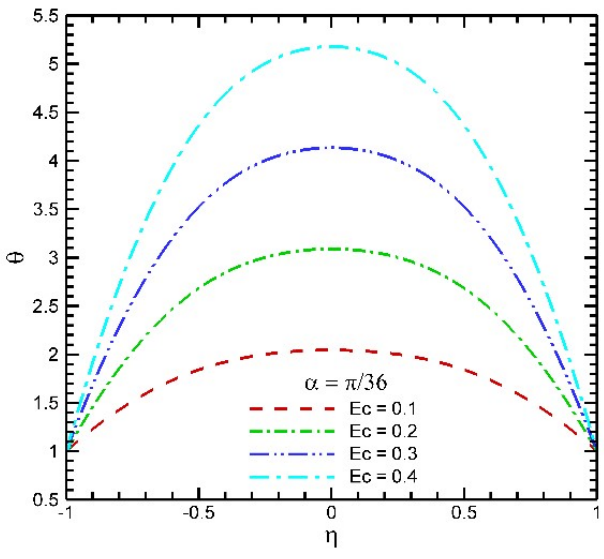

(a)

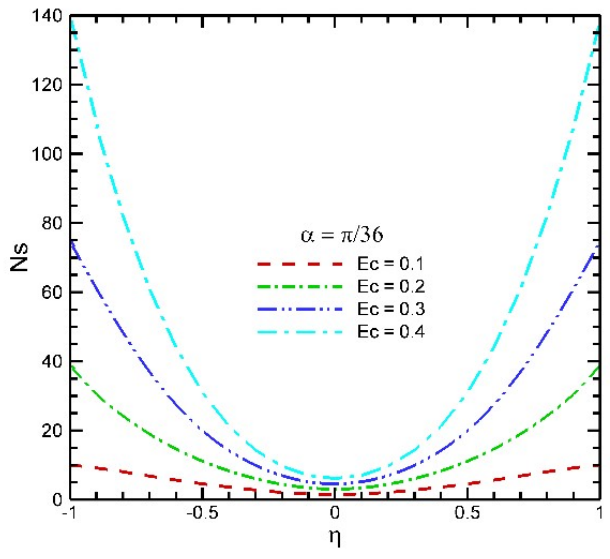

(b)

Figure 7: (a) Temperature distribution (b) Entropy generation for different values of $E c$ at

$$
\alpha=\pi / 36, \phi=0.07, \operatorname{Re}=20, \operatorname{Pr}=6.2, H a=10
$$

The effect of the Eckert number on temperature distribution and the entropy generation for the divergent channel is demonstrated in Figure 7(a) and 7(b) respectively. The heat transfer rate of the nanofluid gains the maximum level at the centerline of the channel which is plotted in Figure 7(a). It is clear that there also exists an increasing trend of increasing Eckert number. The increasing value of $E c$ results in the fastest rate of temperature distribution. Entropy generation for various Eckert number is also presented in Figure 7(b). Here, it can be noticed that at the centerline of the channel the entropy decreases significantly with the increasing value of the Eckert number. Highest $E c$ generates the highest entropy with irreversibility for the nanofluid. 


\subsection{Converging Channel}

The effect of various physical and thermal parameter for the converging channel on velocity profiles and heat transfer rate are represented graphically and discussed here.

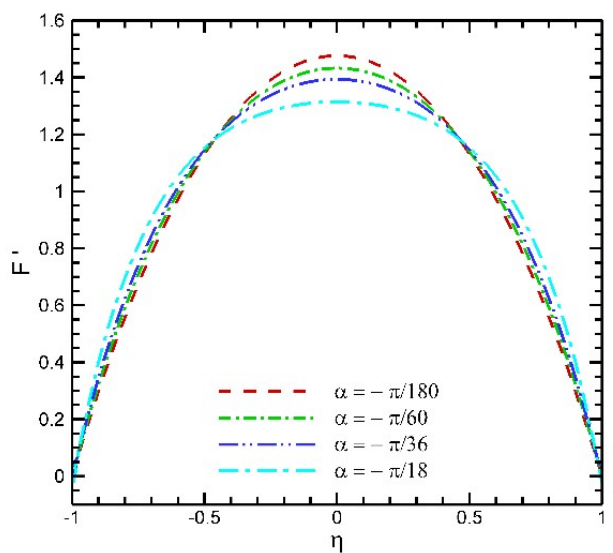

(a)

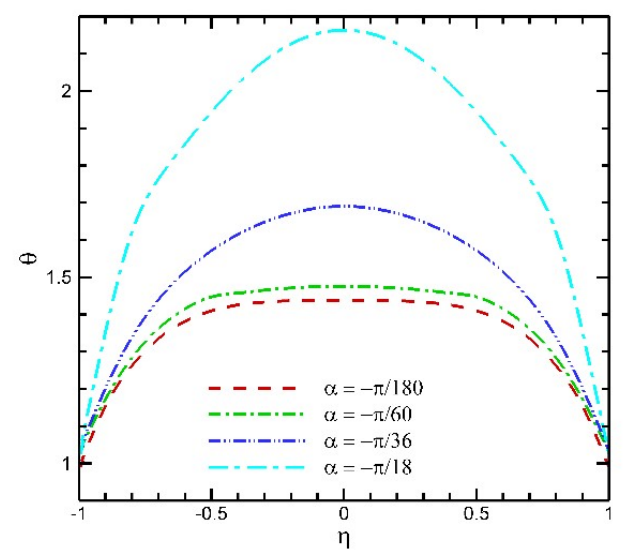

(b)

Figure 8: (a) Velocity profile (b) Temperature distribution for different values of $\alpha$ at $H a=10, \phi=0.07, \operatorname{Re}=20, \operatorname{Pr}=6.2, E c=0.1$

The possession of channel angle for the converging channel on velocity profiles and temperature distribution are represented graphically in Figure 8(a) and 8(b), respectively. From Figure 8(a), it can be noticed that in the convergent channel the velocity of the fluid descends for the accelerating values of the channel closing angle. The heat transfer rate in this channel is also presented in Figure 8(b). Here, it is immersing to notice that an antipodean effect created for the temperature at the centerline of the channel. Convergent channel angle $-\pi / 180$ exhibits the highest temperature for the nanofluid. On the contrary, the smallest heat transfer rate can be exposed to $-\pi / 18$ in the channel.

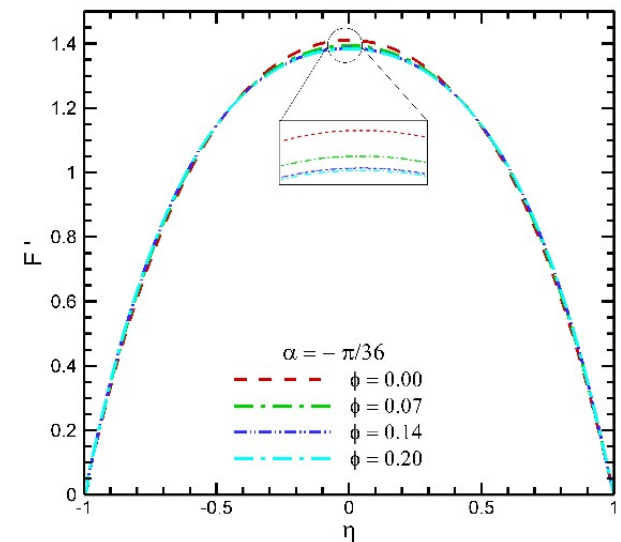

(a)

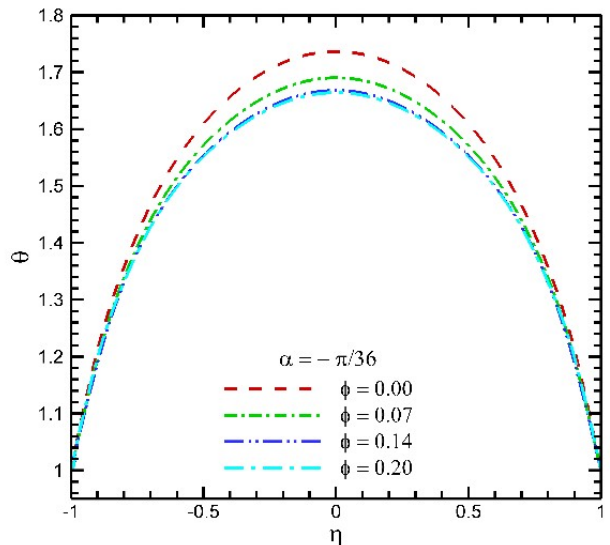

(b)

Figure 9: (a) Velocity profile (b) Temperature distribution for different values of $\phi$ at $\alpha=-\pi / 36, H a=10, \operatorname{Re}=20, \operatorname{Pr}=6.2, E c=0.1$

The effect of nanoparticle volume friction for the convergent channel is plotted in the above figures. Figure 9(a) denotes the velocity profiles and Figure 9(b) denotes the temperature distribution for the convergent channel. It can be noted here that for the expanding amount of volume friction the velocity of the fluid shows the reverse conclusion. Only the base fluid outcomes the highest velocity and base fluid with $\mathrm{Cu}$-nanoparticles show the less. Nanofluid of $20 \%$ nanoparticles terminates the lowest velocity for the convergent channel. The rate of heat transfer with the same thermo physical properties are presented in Figure 9(b). Here the base fluid also generates 
the highest temperature in the centerline of the channel and then the nanofluid with $C u$-nanoparticles of different volume friction.

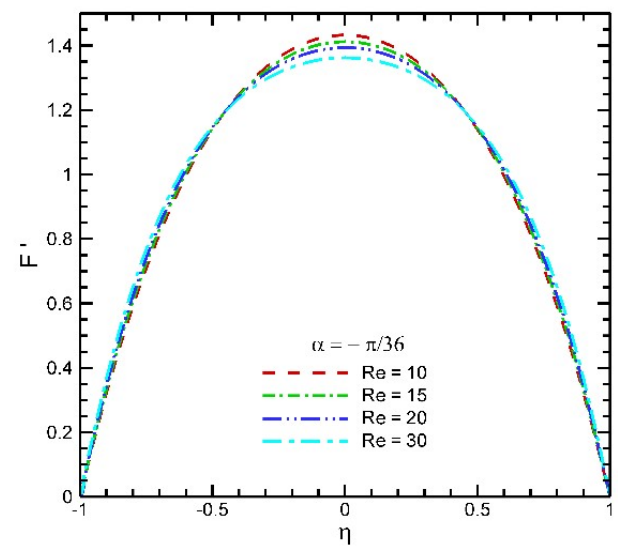

(a)

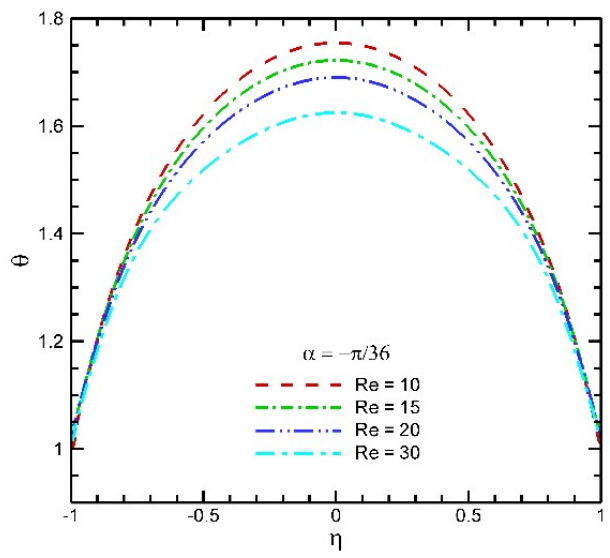

(b)

Figure 10: (a) Velocity profile (b) Temperature distribution for different values of $\operatorname{Re}$ at $\alpha=-\pi / 36, H a=10, \phi=0.07, \operatorname{Pr}=6.2, E c=0.1$

Figure 10(a)-10(b) represent the influence of Reynolds number in the convergent channel on velocity profiles and temperature distribution. In Figure 10(a) it is seen that the rising fluid flow results in less velocity. Nanoparticles volume friction is kept fixed at 0.07 in this case. In this investigation Reynolds number 30 causes the lowest velocity at the centerline. The heat transfer rate behaves the same characteristic for the same thermo physical properties. Also, the highest fluid flow generates the lowest temperature in the convergent channel which can be cleared from Figure 10(b). Here the centerline temperature also rises high compared to the temperature of the channel walls. Also, the same growing effect of increasing Reynolds number can be explored for the heat transfer rate.

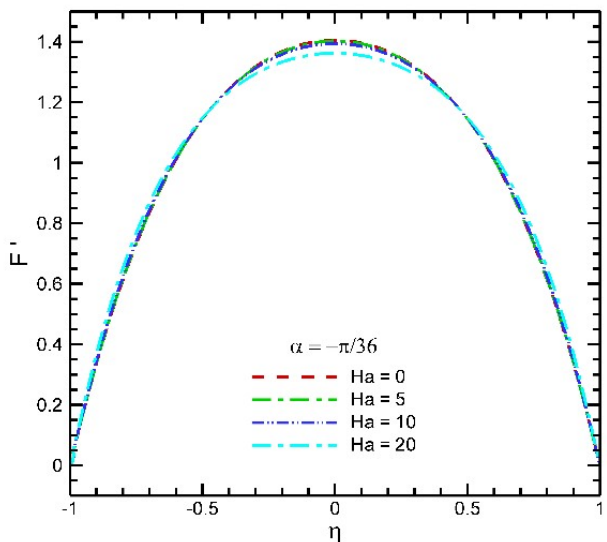

(a)

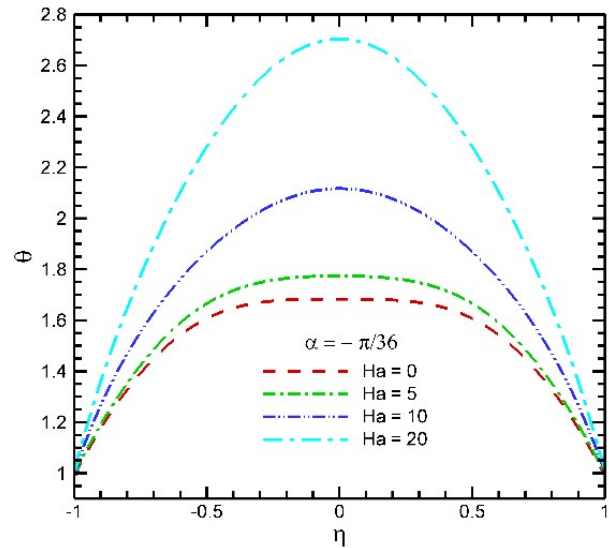

(b)

Figure 11: (a) Velocity profile (b) Temperature distribution for different values of $H a$ at $\alpha=-\pi / 36, \operatorname{Re}=20, \phi=0.07, \operatorname{Pr}=6.2, E c=0.1$

Figure 11(a)-(b) stands for the effect of the Magnetic Hartmann number on the velocity profiles and the temperature profiles for the convergent channel. From the above Figure 11(a), it can be observed that the Hartmann number has a very minimal effect on the velocity profiles for the convergent channel. In this case, the flow rate of the nanofluid reduces because of the rising magnetic field in the channel. The velocity reaches the highest level at the centerline of the channel. But the velocity has the lowest performance at the two walls of the channel for the convergent channel. The temperature distribution is seen in Figure 11(b). For the heat transfer rate, the magnetic Hartmann number has an opposite characteristic compared to the velocity profiles. The 
highest temperature is recorded for the highest $\mathrm{Ha}$ among all $\mathrm{Ha}$ which has been used in this study. This shows that $H a=20$ exhibits the highest temperature and $H a=0$ exhibits the lowest. Here, it is also significant that the temperature rises in the centerline of the channel.

\section{CONCLUSIONS}

In the current study, we have investigated the domination of $\mathrm{Cu}$-nanoparticles on the entropy generation in the magnetohydrodynamic flow of viscous incompressible fluid. Here the water has been used as the base fluid. The effects of various physical parameters on the entropy generation with velocity field and temperature distribution are discussed in detail with the graphical representation. Based on the obtained tabular and graphical results the main inferences of this study are as follows:

- At the two walls, in the solution of the shear stress, two bifurcations at the critical angle are found namely the upper branch and lower branch. In this investigation, it has been initiated that with the increases in nanoparticles volume friction, the value of skin friction coefficient also amplifies.

- Enlargement in channel opening causes increasing in entropy generation, velocity and temperature distribution for both convergent and divergent channel but the fluid flow has an opposite behavior for the convergent channel.

- Significantly, there is a conflicting effect on the increasing nanoparticles volume friction between the convergent and divergent channel.

- The Reynolds number has an increasing influence with the increment of Re for the divergent channel and an opposite effect is observed for the convergent channel.

- The velocity profiles at the centerline against Hartmann number has a rising effect but the temperature distribution has the reversed characteristics in both channels.

- The behavior of the Entropy generation for the Eckert number has an increasing effect at the centerline of the channel and the heat transfer rate has the same for the divergent channel.

\section{REFERENCES}

Das S.K., Choi S.U.S., Yu W. and Pradeep P.T., 2007. Nanofluids: Science and Technology, Wiley.

Choi S., 1995. Enhancing thermal conductivity of fluids with nanoparticles, In Proceedings of the 1995 ASME International Mechanical Engineering Cong and Exposition, San Francisco, USA, 66, 99-105.

Hamadiche M., Scott J., and Jeandel D., 1994. Temporal stability of Jeffery-Hamel flow, Journal of Fluid Mechanics, 268, 71-88.

Jeffery G., 1915. The two -dimensional steady motion of a viscous fluids, Phil. Mag, 6, 455-465.

Hamel G., 1916. Spiralformige Bewgungen Zaher Flussigkeiten, Jahresbericht der Deutschen Math, 25, 34-60.

Fraenkel L.E., 1962. Laminar flow in symmetrical channels with slightly curved walls. I: On the Jeffery-Hamel solutions for flow between plane walls, Proceedings of the Royal Society, 267, 119-138.

Jeong Y.H., Chang W.J. and Chang S.H., 2008. Wettability of heated surfaces under pool boiling using surfactant solutions and nano-fluids, International Journal of Heat and Mass, 51(11-12), 3025-3031.

Aoki K., Baranger C., Hattori M. and Kosuge S., 2017. Slip Boundary Conditions for the Compressible NavierStokes Equations, Journal of Statistical Physics, Springer Verlag, 4, 744-781.

Sobey I.J. and Drazin P.G., 1986. Bifurcation of two-dimensional channel flows, Journal of Fluid Mechanics, 171, 263-287.

Moradi A., Alsaedi A. and Hayat T., 2015. Investigation of heat transfer and viscous dissipation effects on the Jeffery-Hamel flow of nanofluids, Thermal Science, 19(2), 563-578.

Pak B.C. and Cho Y.I., 1998. Hydrodynamic and heat transfer study of dispersed fluids with submicron metallic oxide particles, Experimental Heat Transfer, 11, 151-170.

Seth G.S. and Singh M.K., 2008. Combined free and convection MHD flow in a rotating channel with perfectly conducting wall, Indian Journal of Theoretical Physics, 56, 203-222.

Bejan A., 1996. Entropy generation minimization, CRC Press, New York.

Alam Md.S., Hakim M.A.H. and Makinde O.D., 2017. Magneto-nanofluid dynamics in convergent-divergent channel and its inherent irreversibility, Defect and Diffusion Forum, 377, 95-110.

Aminossadati S.M. and Ghasemi B., 2007. Natural convection cooling of a localized heat source at the bottom of a nanofluid filled enclosure, European Journal of Mechanics-B/Fluids, 9, 630-640.

Das S., Chakraborty S., Jana R.N. and Makinde, O.D., 2005. Entropy analysis of unsteady magneto-nanofluid flow past accelerating stretching sheet with convective boundary condition, Applied Mathematics and Mechanics, 36(12), 1593-1610. 
Padé H., 1892. Sur la représentation approchée d'une fonction pourdes fractions rationnelles, Annales Scientifiques. I’École Normale Supérieure, 9(3), 1-93.

Hermite C., 1893. Sur la généralisation des fractions continues algébriques, Annali di Mathematica Pura e Applicata, 21(2), 289-308.

Khan M.H.K., 2002. High-Order Differential Approximants, Journal of Computational and Applied Mathematics, 149, 457-468.

Rahman M.M., 2004. A New Approach to Partial Differential Approximants [M. Phil thesis], Bangladesh University of Engineering \& Technology, Dhaka.

Drazin P.G. and Tourigny Y., 1996. Numerically study of bifurcation by analytic continuation of a function defined by a power series, SIAM Journal of Applied Mathematics, 56, 1-18.

(C) 2021 the Authors. Journal of Engineering Science published by Faculty of Civil Engineering, Khulna University of Engineering \& Technology. This is an open access article under the terms of the Creative Commons AttributionNonCommercial-NoDerivatives License, which permits use and distribution in any medium, provided the original work is properly cited, the use is non-commercial and no Modifications or adaptations are made. 